\title{
Anomalous aggregation state of deuterium molecules in the nanoscale pores of a metal organic framework
}

\section{$\operatorname{AUTHOR}(\mathrm{S})$ :}

Kanoya, Izuru; Furuta, Terumi; Sakamoto, Ryogo; Hosoe, Mitsuya; Ichikawa, Masao; Itoh, Keiji; Fukunaga, Toshiharu

\section{CITATION:}

Kanoya, Izuru ...[et al]. Anomalous aggregation state of deuterium molecules in the nanoscale pores of a metal organic framework. JOURNAL OF APPLIED PHYSICS 2010, 108(7): 074310.

\section{ISSUE DATE:}

2010-08

URL:

http://hdl.handle.net/2433/147192

\section{RIGHT:}

Copyright 2010 American Institute of Physics. This article may be downloaded for personal use only. Any other use requires prior permission of the author and the American Institute of Physics. The following article appeared in JOURNAL OF APPLIED PHYSICS108, 074310 (2010) and may be found at 


\title{
Anomalous aggregation state of deuterium molecules in the nanoscale pores of a metal organic framework
}

\author{
Izuru Kanoya, ${ }^{1, \text { a) }}$ Terumi Furuta, ${ }^{1}$ Ryogo Sakamoto, ${ }^{1}$ Mitsuya Hosoe, ${ }^{1}$ Masao Ichikawa, ${ }^{1}$ \\ Keiji Itoh, ${ }^{2}$ and Toshiharu Fukunaga ${ }^{2}$ \\ ${ }_{1}^{1}$ Fundamental Technology Research Center, Honda R\&D Co., Ltd., Wako, Saitama 351-0193, Japan \\ ${ }^{2}$ Research Reactor Institute, Kyoto University, Kumatori, Osaka 590-0494, Japan
}

(Received 8 April 2010; accepted 23 August 2010; published online 13 October 2010)

\begin{abstract}
The behavior of hydrogen molecules adsorbed onto the nanospace of porous materials is of great interest but is poorly understood. Here we show direct visualization of deuterium molecules adsorbed on isoreticular metal organic frameworks (IRMOF-1) at $77 \mathrm{~K}$ using reverse Monte Carlo simulation based on first principle molecular dynamics simulation and neutron diffraction. Results show that the two types of adsorption sites around a $\mathrm{ZnO}_{4}$ cluster are almost fully occupied by the deuterium molecules but that other sites are not fully occupied. Moreover an interesting information about the aggregation state of deuterium molecules was directly obtained from the deuteriumdeuterium partial pair distribution function. Namely, the average distance of deuterium molecules adsorbed onto IRMOF-1 is slightly longer than that in a solid state but much shorter than that in the corresponding gas state. (C) 2010 American Institute of Physics. [doi:10.1063/1.3490625]
\end{abstract}

\section{INTRODUCTION}

Hydrogen shows promise as a next-generation energy source from the perspective of $\mathrm{CO}_{2}$ emissions and renewal energy. Various kinds of hydrogen storage materials have been examined for the realization of small and lightweight hydrogen storage systems. Porous materials are the most common potential candidates as hydrogen storage materials because they can physically adsorb hydrogen molecules under a low heat of adsorption. Recently, metal organic frameworks (MOFs), which are formed by metal oxide clusters with organic linkers, have attracted a great deal of interest lately because of their large surface areas. ${ }^{1}$ In particular, isoreticular MOFs (IRMOFs) have been investigated by a number of researchers with respect to their hydrogen (deuterium) adsorption properties. ${ }^{2-5}$ Rosi et $a l^{2}$ reported that IRMOF compounds are capable of adsorbing a large amount of hydrogen at $78 \mathrm{~K}$ and room temperature. They also reported an inelastic neutron scattering spectra for adsorbed hydrogen in IRMOF-1 (MOF5), whose structure is shown in Fig. 1, and showed that sites around the zinc oxide cluster and the 1,4-benzenedicarboxylate (BDC) hexagonal linker are available during hydrogen adsorption. Later, Yildirim and Hartman $^{4}$ performed neutron diffraction measurements at 3.5 $\mathrm{K}$ and reported the presences of four deuterium adsorption sites. Namely, three of them are different sites around the zinc oxide cluster and these sites are termed Cup site, $\mathrm{ZnO}_{3}$ site, and $\mathrm{ZnO}_{2}$ site, respectively. The other site is the top of the hexagonal linker, which was termed Hex site. ${ }^{4}$ However, these measurements were performed below the solidification temperature for deuterium. From a practical application standpoint it is necessary to determine the adsorption sites in their temperature range for gaseous adsorption. In this work, therefore, the deuterium adsorption sites in IRMOF-1 were investigated at $77 \mathrm{~K}$ using a pair distribution function tech-

${ }^{a)}$ Electronic mail: izuru_kanoya@n.f.rd.honda.co.jp. nique derived from the Fourier transformation of a structure factor obtained by neutron diffraction. The pair distribution function directly provides the correlation distances between atoms but only yields one-dimensional information. Therefore, reverse Monte Carlo (RMC) modeling, ${ }^{6}$ a wellestablished and effective method of constructing threedimensional atomic arrangements of crystalline and disordered materials, and molecular dynamics simulation were used in order to determine the adsorption sites.

The results show interesting information about not only the adsorption sites but also the aggregation state of $\mathrm{D}_{2}$ molecules adsorbed onto IRMOF-1 under $1 \mathrm{MPa} \mathrm{D}_{2}$ pressure. That is, unusually-short $\mathrm{D}_{2}-\mathrm{D}_{2}$ distance, which corresponds to that in a gas state under about 50 -fold pressure, is observed in the deuterium-deuterium partial pair distribution function.

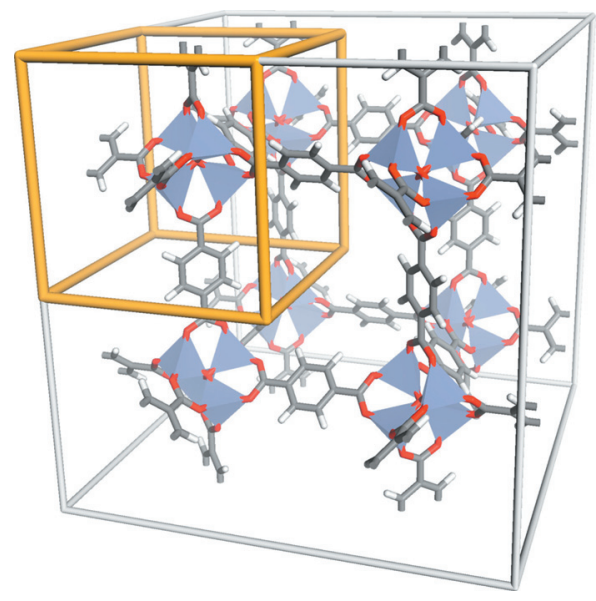

FIG. 1. (Color) Structure of IRMOF-1 (Ref. 16). The small cube corresponds to the unit structure in Fig. 7(a). 


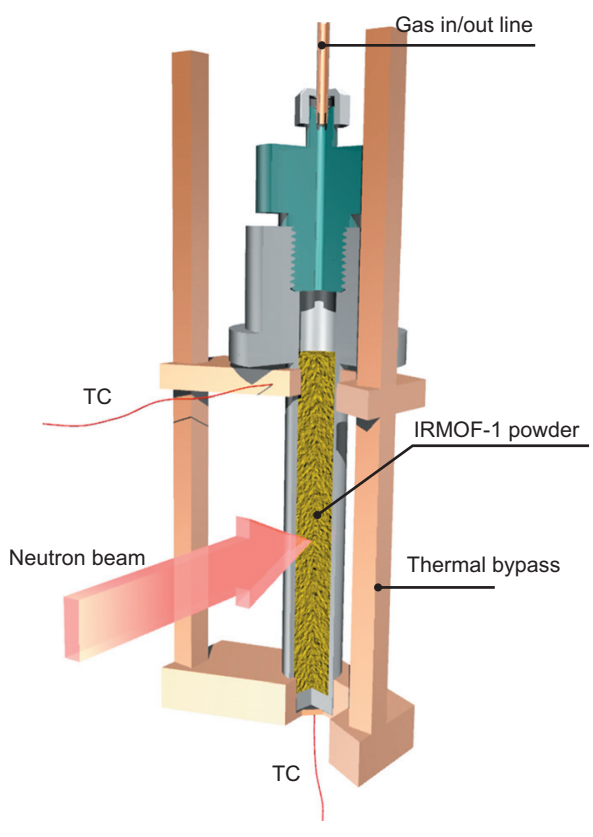

FIG. 2. (Color) Schematic diagram of container for neutron diffraction measurements.

\section{EXPERIMENTAL PROCEDURE}

\section{A. Sample preparation}

A deuterated IRMOF-1 sample was prepared in the same way as reported in Ref. 16. That is, a mixture of $\mathrm{Zn}\left(\mathrm{NO}_{3}\right)_{2} \cdot 4 \mathrm{H}_{2} \mathrm{O}$ and deuterated $\mathrm{BDC}$ was dissolved in a distilled $\mathrm{N}, \mathrm{N}^{\prime}$-diethylformamide, and the solution was heated at $353 \mathrm{~K}$ for $24 \mathrm{~h}$ in a closed vessel. A crystalline d-IRMOF-1 powder was obtained in $90 \%$ yield.

\section{B. Neutron diffraction experiments}

The neutron diffraction measurements were carried out in the high intensity total scattering spectrometer installed at the pulsed neutron source of the High Energy Accelerator Research Organization (KEK, Tsukuba, Japan). A pressureresistant cylindrical container made of $\mathrm{Ti}-\mathrm{Zr}$ neutron zeroscattering alloy with an inner diameter of $8.0 \mathrm{~mm}$ and a wall thickness $1.0 \mathrm{~mm}$ was used. A schematic diagram of the container is shown in Fig. 2. The top of the container was cooled using a helium cryostat. A thermal bypass made of copper was attached to the top and bottom of the container in order to reduce the temperature differential between them. Two thermocouple sensors were located at the top and bottom of the container and the maximum temperature difference was within $3 \mathrm{~K}$. Deuterium gas was loaded through a $1 / 16$ in. external diameter stainless steel tube that was connected to the top of the container.

The neutron diffraction measurements were performed on the IRMOF-1 powder sample at $77 \mathrm{~K}$ under vacuum and $1 \mathrm{MPa} \mathrm{D}_{2}$ pressure. Under the latter condition, the scattering from $\mathrm{D}_{2}$ gas going into the spaces between the powder particles is not negligible. Thus the scattering intensity of $\mathrm{D}_{2}$ gas under $1 \mathrm{MPa}$ pressure was also measured at $77 \mathrm{~K}$ and subtracted from the scattering intensity of the IRMOF-1 powder measured under $1 \mathrm{MPa} \mathrm{D}_{2}$ pressure to account for the filling factor of the IRMOF-1 powder. The amount of deuterium adsorption in the IRMOF-1 sample was evaluated from the pressure change, and the deuterium molecule to $\mathrm{Zn}$ ratio was determined to be $\mathrm{D}_{2} / 4 \mathrm{Zn}=16.0(1)$, which agrees well with that obtained by a Sieverts' type apparatus.

The structure factors, $S(Q)$, where $Q=4 \pi \sin \theta / \lambda(2 \theta$ is the scattering angle and $\lambda$ the neutron wavelength), were derived by the application of various corrections for the background absorption ${ }^{7}$ and multiple scattering, ${ }^{8}$ as well as via normalization using a vanadium rod.

$S(Q)$ was analyzed by the Faber-Ziman definition ${ }^{9}$ as follows:

$$
S(Q)=\frac{I(Q)-\left\{\left\langle b^{2}\right\rangle-\langle b\rangle^{2}\right\}}{\langle b\rangle^{2}},
$$

and

$$
\left\langle b^{2}\right\rangle=\sum_{\mathrm{i}} c_{i} b_{i}^{2}, \quad\langle b\rangle=\sum_{\mathrm{i}} c_{i} b_{i},
$$

where $c_{i}$ and $b_{i}$ are the concentration and the coherent scattering length for neutron diffraction of the component atoms $i$, respectively. The pair distribution function, $g(r)$, can be derived from the Fourier transformation of $S(Q)$ as follows:

$$
\mathrm{g}(r)=1+\frac{1}{2 \pi^{2} r \rho} \int_{0}^{Q_{\min }} Q(S(Q)-1) \sin Q r d Q,
$$

where $\rho$ is the average number density of the atoms.

The $g(r)$ for an IRMOF1 compound is written as a weighted sum of ten partial pair distribution functions, $g_{i-j}(r)$ :

$$
g(r)=\sum_{i, j} w_{i-j} g_{i-j}(r) .
$$

Here $w_{i-j}$ is the weighting factor, defined as:

$$
w_{i-j} \frac{c_{i} c_{j} b_{i} b_{j}}{\langle b\rangle^{2}} .
$$

Due to the deuterium adsorption, the number of partial pair distribution functions is fatally increased to 15 . However, only the pair correlations related to adsorbed deuterium molecules $\left(D^{\text {ad }}-\mathrm{Zn}, \mathrm{D}^{\mathrm{ad}}-\mathrm{O}, \mathrm{D}^{\mathrm{ad}}-\mathrm{C}\right.$, and $\mathrm{D}^{\mathrm{ad}}-\mathrm{D}^{\text {hex }}$ and $\mathrm{D}^{\mathrm{ad}}-\mathrm{D}^{\mathrm{ad}}$ pairs, here $\mathrm{D}^{\text {ad }}$ stands for adsorbed deuterium molecules and $\mathrm{D}^{\text {hex }}$ for deuterium atoms belonging to the hexagonal linker in IRMOF-1) were required to investigate the $\mathrm{D}_{2}$ adsorption sites in the IRMOF-1 compound. Sum of the weighting factors of these pair correlations are 0.625 for the IRMOF-1 powder measured under $1 \mathrm{MPa} \mathrm{D}_{2}$ pressure. The other 0.375 is a constituent of the host IRMOF-1, and then we can remove its contribution and obtain a pair distribution function related to adsorbed deuterium molecules, $g^{\mathrm{D}_{2}}(r)$, as follow:

$$
\begin{aligned}
g^{\mathrm{D}_{2}}(r)= & {\left[g(r)\left(\text { at } 1 \mathrm{MPa} \mathrm{D}_{2}\right)-0.375\right.} \\
& \times g(r)(\text { under vacuum })] \times 1.6 .
\end{aligned}
$$



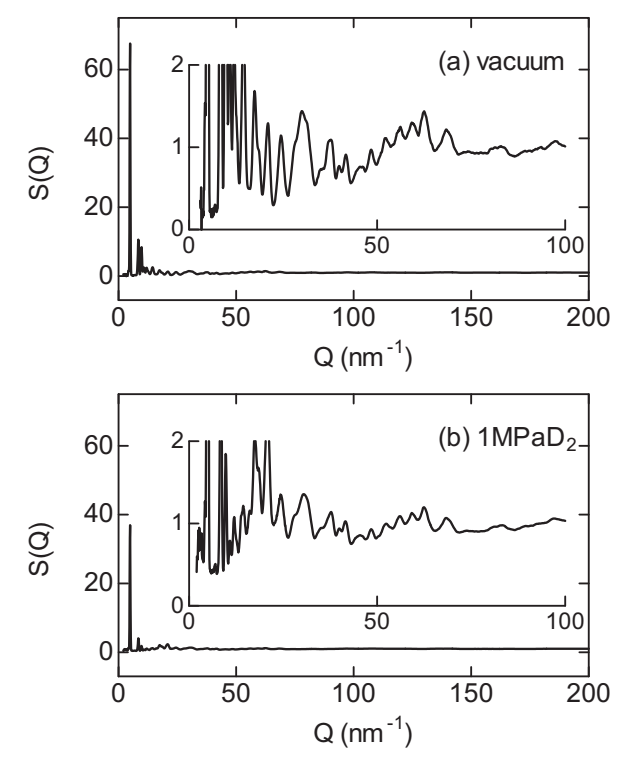

FIG. 3. Total structure factors, $\mathrm{S}(Q)$, for the IRMOF1 powder measured at $77 \mathrm{~K}$ (a) under vacuum and (b) at $1 \mathrm{MPa} \mathrm{D}_{2}$ pressure covering the full $Q$-range, and enlarged illustrations in the region below $100 \mathrm{~nm}^{-1}$.

\section{RESULTS AND DISCUSSION}

\section{A. Neutron diffraction}

Figure 3 shows the total structure factors, $S(Q)$, for the IRMOF-1 powder measured at $77 \mathrm{~K}$ under vacuum and at 1 MPa deuterium pressure. The pair distribution functions, $g(r)$, which were derived from the Fourier transformation of these total structure factors with a $Q_{\max }$ of $200 \mathrm{~nm}^{-1}$, are compared in Fig. 4. Here the $g(r)$ curve for the IRMOF-1 powder measured under vacuum is multiplied by the factor 0.375 for comparison. Both $g(r)$ curves have two peaks at $0.107 \mathrm{~nm}$ and $0.136 \mathrm{~nm}$ and these peaks mainly correspond to $\mathrm{C}-\mathrm{D}^{\text {hex }}, \mathrm{C}-\mathrm{O}$, and $\mathrm{C}-\mathrm{C}$ correlations, respectively. On the other hand, a new sharp peak is observed at $0.07 \mathrm{~nm}$ in the $g(r)$ curve after deuterium loading that corresponds to $\mathrm{D}^{\text {ad }}-\mathrm{D}^{\text {ad }}$ correlations. The coordination number, calculated from the area under the peak, was 1.0(3). This result indicates that all deuterium adsorbed onto IRMOF-1 are in the form of molecules. Moreover, difference in the $g(r)$ curves before and after the deuterium loading can be seen above $0.25 \mathrm{~nm}$ and these correspond to the pair correlations related

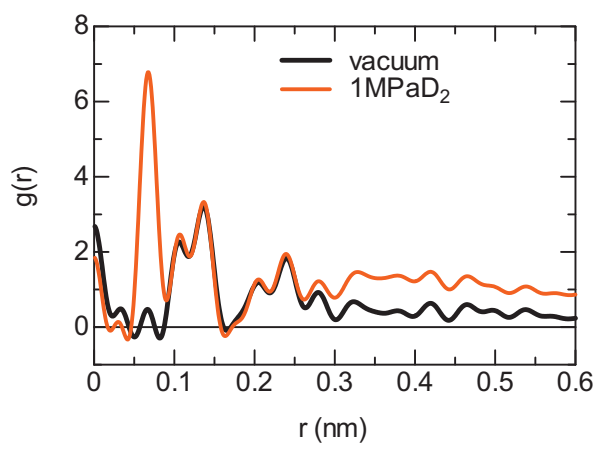

FIG. 4. (Color) Pair distribution functions, $g(r)$, for the IRMOF1 powder measured at $77 \mathrm{~K}$ under vacuum and at $1 \mathrm{MPa}_{2}$ pressure. The $g(r)$ curve for the IRMOF1 powder measured under vacuum is multiplied by the factor 0.375 for comparison.

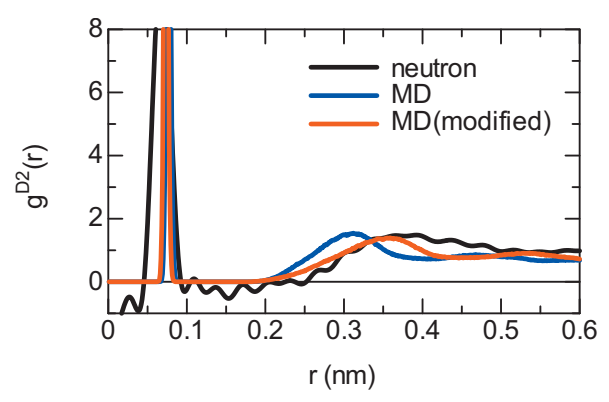

FIG. 5. (Color) The pair distribution function related to the adsorbed $\mathrm{D}_{2}$ molecules, such as $\mathrm{D}^{\text {ad }}-\mathrm{Zn}, \mathrm{D}^{\mathrm{ad}}-\mathrm{O}, \mathrm{D}^{\mathrm{ad}}-\mathrm{C}$, and $\mathrm{D}^{\text {ad }}-\mathrm{D}^{\text {hex }} \mathrm{D}^{\text {ad }}-\mathrm{D}^{\text {ad }}$ correlations, obtained by using Eq. (6) (black line). Neutron-weighted sum of partial pair distribution functions related to $\mathrm{D}_{2}$ molecules $\left(\mathrm{D}^{\mathrm{ad}}-\mathrm{Zn}, \mathrm{D}^{\mathrm{ad}}-\mathrm{O}\right.$, $\mathrm{D}^{\text {ad }}-\mathrm{C}, \mathrm{D}^{\text {ad }}-\mathrm{D}^{\text {hex }}$, and $\mathrm{D}^{\text {ad }}-\mathrm{D}^{\text {ad }}$ correlations) as calculated by $\mathrm{MD}$ simulation (blue and red lines).

to the adsorbed $\mathrm{D}_{2}$ molecules, such as $\mathrm{D}^{\mathrm{ad}}-\mathrm{Zn}, \mathrm{D}^{\text {ad }}-\mathrm{O}$, $\mathrm{D}^{\text {ad }}-\mathrm{C}$, and $\mathrm{D}^{\text {ad }}-\mathrm{D}^{\text {hex }} \mathrm{D}^{\text {ad }}-\mathrm{D}^{\text {ad }}$ correlations. The pair distribution function related to adsorbed deuterium molecules, $g^{\mathrm{D}_{2}}(r)$, which was obtained by using Eq. (6), is presented in Fig. 5.

\section{B. First principle molecular dynamics (FPMD)}

The neutron diffraction results directly give the precise pair correlations between $\mathrm{D}_{2}$ molecules and constituent atoms of IRMOF-1. However, additional information is needed in order to elucidate the deuterium adsorption sites because the neutron pair distribution function is one-dimensional information. We performed FPMD simulations based on density functional theory (DFT) (Ref. 10) local density approximations ${ }^{11,12}$ in order to obtain information about adsorption states (such as dissociation of molecular) as well as adsorption sites. In the FPMD simulations, a super cell with $\mathrm{a}=\mathrm{b}=\mathrm{c}=1.8151 \mathrm{~nm}$ and $\alpha=\beta=\gamma=60^{\circ}$ was used, and 106 atoms (Zn: 8, O: $\left.26, \mathrm{C}: 48, \mathrm{D}^{\text {hex }}: 24\right)$ were located at the positions reported by Eddaoudi et al. ${ }^{16}$ The deuterium molecules $\left(32\right.$ molecules, $\left.\mathrm{D}_{2} / 4 \mathrm{Zn}=16.0\right)$ were put randomly in the unit cell as a gas phase. The FPMD simulations were performed using the projector augmented-wave ${ }^{15}$ method calculation package, VASP, ${ }^{13,14,21,27}$ with $1 \times 1 \times 1$ k-point meshes. Here, the atomic weight of hydrogen atoms was changed to 2.014 for them to become deuterium atoms. The plane wave energy cut-off and convergence energy conditions were determined as $400 \mathrm{eV}$ and $0.1 \mathrm{meV}$, respectively. The FPMD calculations were performed using NVT (the constant entity-number/volume/temperature) ensemble at 77 $\mathrm{K}$ for more than 35000 steps with an integral time of $0.2 \mathrm{fs}$. To obtain an average result, FPMD calculations were executed five times using different initial configurations of deuterium molecule positions and over 200000 total MD steps were used to analysis.

The partial pair distribution functions related to the deuterium molecules $\left(\mathrm{D}^{\mathrm{ad}}-\mathrm{Zn}, \mathrm{D}^{\mathrm{ad}}-\mathrm{O}, \mathrm{D}^{\mathrm{ad}}-\mathrm{C}, \mathrm{D}^{\mathrm{ad}}-\mathrm{D}^{\text {hex }}\right.$, and $\mathrm{D}^{\text {ad }}-\mathrm{D}^{\text {ad }}$ correlations) were calculated using the configuration of deuterium molecules. Here, each correlation peak was broadening using Gaussian function with $\sigma=0.05$. The neutron-weighted sum of these functions is compared in Fig. 5 (blue line) with the experimental results. The difference at around $0.3-0.4 \mathrm{~nm}$ between two curves is thought to be due 

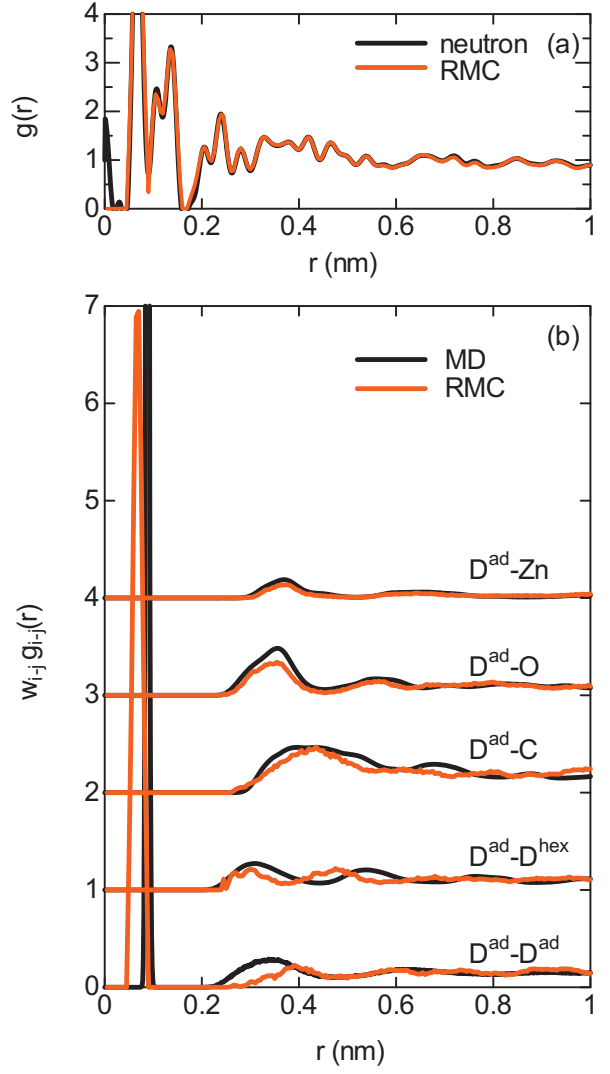

FIG. 6. (Color) (a) Pair distribution functions, $g(r)$, observed by neutron diffraction for IRMOF-1 measured at $1 \mathrm{MPa}_{2}$ pressure, compared with the neutron diffraction $g(r)$ functions calculated from the RMC model (red line). (b) Partial pair distribution functions $g(r)$ calculated from the MD, compared with those calculated from the RMC model (red lines).

to an over-binding of the hydrogen adsorption energies based on DFT calculations. Because the hydrogen adsorption energy of benzene based on DFT was $9.75 \mathrm{~kJ} / \mathrm{mol}$, which was larger than the experimental value of graphite (4.99 $\mathrm{kJ} / \mathrm{mol}) .{ }^{17}$ Therefore, we needed to correct for the correlation distances of the FPMD results and the correction factor was determined to be 1.15 . The modified function is also plotted in Fig. 5 (red line). Moreover, it can be confirmed that the deuterium molecules do not dissociate but keep molecular state in the adsorption process. This result is agreed with that obtained from $\mathrm{D}^{\text {ad }}-\mathrm{D}^{\text {ad }}$ coordination number of neutron pair distribution function.

\section{RMC simulation}

In order to elucidate the adsorption sites of deuterium molecules in IRMOF, the RMC simulation was applied. RMC simulation was carried out by using a self-written code. A simulation box containing 3392 atoms (Zn: 256, O: 832 , C: $\left.1536, \mathrm{D}^{\text {hex }}: 768\right)$ with periodic boundary conditions was used. First, an RMC configuration of host IRMOF-1 was constructed by fitting the model $g(r)$ to the neutron $g(r)$. Here the crystal structure of IRMOF-1, reported by Eddaoudi et al., ${ }^{16}$ was used as a starting configuration. Maximum movement for each atom was set to $0.002 \mathrm{~nm}$ to keep the initial structure. The neutron $g(r)$ for IRMOF-1, calculated from the RMC model, is plotted by the red line in Fig. 6 . (a)

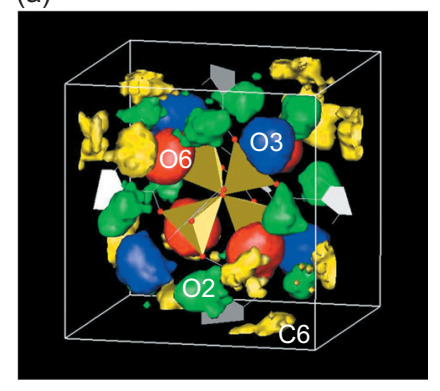

(b)

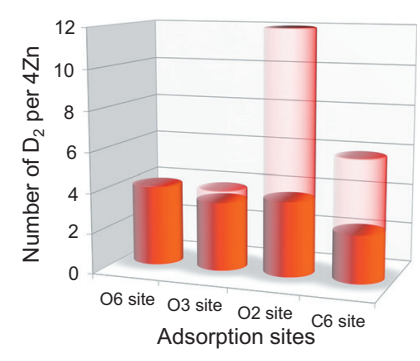

FIG. 7. (Color) (a) Isosurface plots of deuterium molecules adsorbed on the IRMOF-1 derived from the RMC configuration. The average density distribution of deuterium molecules was divided into 0.05-nm-sided cubes. (b) The occupancy rates for deuterium molecules at each adsorption site. Four, 4, 12, and 4 sites per $4 \mathrm{Zn}$ for $\mathrm{O} 6, \mathrm{O} 3$, and $\mathrm{O} 2$, respectively.

Next, the adsorption sites of $\mathrm{D}_{2}$ molecules were determined. The previously constructed configuration of $\mathrm{Zn}, \mathrm{O}, \mathrm{C}$, and $\mathrm{D}^{\text {hex }}$ atoms in IRMOF-1 was used, and these atoms were fixed in the RMC simulation. Namely, only deuterium molecules were moved. Recently, not only experimental data but also classical molecular dynamics simulation results are used in order to improve the reliability of the RMC modeling. ${ }^{22-26}$ Here the RMC simulation was performed referencing the FPMD simulation result. Namely, the configuration of deuterium molecules, obtained by FPMD simulation, was used as an initial configuration. Moreover, the RMC model was fitted not only to the neutron $g(r)$ but also to the partial pair distribution functions obtained from the FPMD simulation results. The closest distances between two atoms $\left(\mathrm{D}^{\mathrm{ad}}-\mathrm{Zn}\right.$, $\mathrm{D}^{\text {ad }}-\mathrm{O}, \mathrm{D}^{\mathrm{ad}}-\mathrm{C}$, and $\mathrm{D}^{\text {ad }}-\mathrm{D}^{\text {hex }}$ ) were determined to be 0.25 nm.

The total and partial $g(r)$ curves, calculated from the RMC model, are plotted in Fig. 6, and compared with the total $g(r)$ curve obtained by neutron diffraction and the partial pair distribution functions obtained by FPMD simulation. Excellent fits as shown were obtained for the total $g(r)$ curves, along with goodness-fits with the partial $g(r)$ curve.

Figure 7(a) shows the density isosurface plots. Here, the average density distribution of deuterium molecules was divided into $0.05 \mathrm{~nm}$-sided cubes and density isovalue plots $\left(1.5 \times 10^{-5}\right.$ molecules $\left./ \mathrm{nm}^{-3}\right)$ are presented in the figure. Four high density regions, indicated by different colors (red, blue, green, yellow), are clearly observed around the $\mathrm{ZnO}_{4}$ cluster and the hexagonal linker. We term these sites the $\mathrm{O} 6$ site, $\mathrm{O} 3$ site, $\mathrm{O} 2$ site, and $\mathrm{C} 6$ site, respectively, based on the $\mathrm{D}^{\mathrm{ad}}-\mathrm{O}$ coordination number. In the previous study, ${ }^{4}$ these sites correspond to the Cup site, $\mathrm{ZnO}_{3}$ site, $\mathrm{ZnO}_{2}$ site, and Hex site, respectively. This result shows that the $\mathrm{O} 6$ and $\mathrm{O} 3$ sites are considerably occupied and that the deuterium molecules are located at these site positions. On the other hand, the $\mathrm{O} 2$ and $\mathrm{C} 6$ sites are comparatively empty and the deuterium molecules are distributed around these site positions. In Fig. 7(b), the occupancies for deuterium molecules in each adsorption site are presented. Indeed, the $\mathrm{O} 6$ and $\mathrm{O} 3$ sites are almost fully occupied by the deuterium molecules. These results are consistent with the calculated adsorption energy for the $\mathrm{O} 6$ and $\mathrm{O} 3$ sites, which are larger than that for $\mathrm{O} 2$ and C6 sites. On the other hand, the $\mathrm{O} 2$ and C6 sites are not fully 
occupied. The occupancy state for $\mathrm{O} 2$ sites is attributable to the interaction between deuterium molecules. That is, the average $\mathrm{O} 6-\mathrm{O} 2$ and $\mathrm{O} 3-\mathrm{O} 2$ distances calculated from the RMC model are $0.36 \mathrm{~nm}$ and $0.34 \mathrm{~nm}$, respectively, which are shorter than the average $\mathrm{D}^{\mathrm{ad}}-\mathrm{D}^{\text {ad }}$ distances. Consequently, repulsion between deuterium molecules is considerable, and they influence the site occupancy of $\mathrm{O} 2$ sites. Therefore, only one-third of the sites are occupied, despite the presence of $\mathrm{O} 2$ sites in large numbers.

The C6 sites are about half empty. Unlike the $\mathrm{O} 2$ sites, the average O6-C6 and O3-C6 distances are more than 0.46 $\mathrm{nm}$ and hence the repulsion between deuterium molecules is weak. Therefore, the occupancy state for C6 sites is thought to be attributable to the relatively-small adsorption energy. Sagara et al. ${ }^{18}$ reported that adsorption energy for C6 sites is $5.27 \mathrm{~kJ} / \mathrm{mol}$. This value is comparable with the experimental value for the hydrogen adsorption energy on graphite (4.99 $\mathrm{kJ} / \mathrm{mol}$ ) (Ref. 17) and smaller than that for other adsorption sites.

The RMC model shows interesting information related to the state of $\mathrm{D}_{2}$ molecules adsorbed onto IRMOF-1. Namely, the $\mathrm{D}^{\mathrm{ad}}-\mathrm{D}^{\mathrm{ad}}$ partial pair distribution function (shown in Fig. 6) has a broad peak around $0.4 \mathrm{~nm}$. This result indicates that the average intermolecular distance of adsorbed deuterium molecules is about $0.4 \mathrm{~nm}$. It is shorter than that in a critical state but close to those in solid states. ${ }^{19,20}$ Such $\mathrm{D}_{2}-\mathrm{D}_{2}$ distance surprisingly corresponds to that in a gas state under about $50 \mathrm{MPa}$ at $77 \mathrm{~K}$ conditions.

\section{CONCLUSIONS}

The adsorption sites of deuterium molecules onto IRMOF-1 were investigated at $77 \mathrm{~K}$, using RMC modeling, based on the associated neutron diffraction data and molecular dynamics simulation. The results show that the $\mathrm{O} 6$ and O3 sites are almost fully occupied by the deuterium molecules but the $\mathrm{O} 2$ and $\mathrm{C} 6$ sites are not. The occupancy state for these sites is dependent not only on the adsorption energy but also on the extent of repulsion between deuterium molecules. Moreover, the results also show that the average distance between deuterium molecules adsorbed onto IRMOF-1 is slightly longer than that in solid phase but shorter than that in a gas state under $1 \mathrm{MPa}$ at $77 \mathrm{~K}$ conditions. Namely, the deuterium molecules are concentrated in a very dense state. Since the motions of deuterium molecules are constrained by the nanospace concurrent with adsorption energy, such irregular states of deuterium molecules occurs in IRMOF-1.
This suggests that the nanoscale control of three-dimensional framework structures allow an increase in the amount of adsorption. We believe strongly that the method used in this study is useful in investigating the behavior of adsorbed molecules.

\section{ACKNOWLEDGMENTS}

We would like to express our sincerest thanks to the Booster Synchrotron Utilization Facility at KEK for providing us the opportunity to conduct our neutron diffraction experiments.

${ }^{1}$ M. Eddaoudi, D. B. Moler, H. Li, B. Chen, T. M. Reineke, M. O'Keeffe, and O. M. Yaghi, Acc. Chem. Res. 34, 319 (2001).

${ }^{2}$ N. L. Rosi, J. Eckert, M. Eddaoudi, D. T. Vodak, J. Kim, M. O'Keeffe, and O. M. Yaghi, Science 300, 1127 (2003).

${ }^{3}$ J. L. C. Rowsell, A. R. Millward, K. S. Park, and O. M. Yaghi, J. Am. Chem. Soc. 126, 5666 (2004).

${ }^{4}$ T. Yildirim and M. R. Hartman, Phys. Rev. Lett. 95, 215504 (2005).

${ }^{5}$ F. M. Mulder, T. J. Dingemans, H. G. Schimmel, A. J. Ramirez-Cuesta, and G. J. Kearley, Chem. Phys. 351, 72 (2008).

${ }^{6}$ R. L. McGreevy and L. Pusztai, Mol. Simul. 1, 359 (1988).

${ }^{7}$ H. H. Paalman and C. J. Pings, J. Appl. Phys. 33, 2635 (1962).

${ }^{8}$ I. A. Blech and B. L. Averbach, Phys. Rev. 137, A1113 (1965).

${ }^{9}$ T. E. Faber and J. M. Ziman, Philos. Mag. 11, 153 (1965).

${ }^{10}$ W. Kohn and L. J. Sham, Phys. Rev. 140, A1133 (1965).

${ }^{11}$ D. M. Ceperley and B. Alder, Phys. Rev. Lett. 45, 566 (1980).

${ }^{12}$ J. P. Perdew and A. Zunger, Phys. Rev. B 23, 5048 (1981).

${ }^{13}$ G. Kresse and J. Hafner, Phys. Rev. B 47, 558 (1993).

${ }^{14}$ G. Kresse and J. Furthmüller, Phys. Rev. B 54, 11169 (1996).

${ }^{15}$ P. E. Blöchl, Phys. Rev. B 50, 17953 (1994).

${ }^{16}$ M. Eddaoudi, J. Kim, N. Rosi, D. Vodak, J. Wachter, M. O'Keeffe, and O. M. Yaghi, Science 295, 469 (2002), and supporting information.

${ }^{17}$ L. Mattera, F. Rosattelli, C. Salvo, F. Tommasini, U. Valbusa, and G. Vidahi, Surf. Sci. 93, 515 (1980).

${ }^{18}$ T. Sagara, J. Klassen, and E. Ganz, J. Chem. Phys. 121, 12543 (2004).

${ }^{19} d_{\text {solid }}(14.15 \mathrm{~K})=0.0763 \mathrm{~g} / \mathrm{cc} . \quad$ dist $_{\mathrm{H}-\mathrm{H}}=0.395 \mathrm{mn} . \quad d_{\text {critical }}(33.25 \mathrm{~K})$ $=0.0310 \mathrm{~g} / \mathrm{cc}$. dist $t_{\mathrm{H}-\mathrm{H}}=0.533 \mathrm{~nm}$.

${ }^{20}$ Encyclopaedia Chimica, ed. Editorial Committee of Encyclopaedia Chimica (Kyoritsu shuppan, Tokyo, 2001), Vol. 5, p. 48 (in Japanese).

${ }^{21}$ G. Kresse and D. Joubert, Phys. Rev. B 59, 1758 (1999).

${ }^{22}$ L. Pusztai, O. Pizio, and S. Sokolowski, J. Chem. Phys. 129, 184103 (2008).

${ }^{23}$ L. Pusztai, I. Harsányi, H. Dominguez, and O. Pizio, Chem. Phys. Lett. 457, 96 (2008).

${ }^{24}$ L. Pusztai, H. Dominguez, O. Pizio, and S. Sokolowski, J. Mol. Liq. 147, 52 (2009).

${ }^{25}$ R. Vácha, T. Megyes, I. Bako', L. Pusztai, and P. Jungwirth, J. Phys. Chem. A 113, 4022 (2009).

${ }^{26}$ V. Mile, L. Pusztai, H. Dominguez, and O. Pizio, J. Phys. Chem. B 113, 10760 (2009).

${ }^{27}$ The FPMD simulations have been performed using the ab-initio totalenergy and molecular dynamics program VASP (Vienna ab-initio simulation program) developed at the Institut fur Material-physik of the Universitat Wien. 